\title{
Risk Factors for Nonsynchronous Second Primary Malignancy and Related Death in Patients with Differentiated Thyroid Carcinoma
}

\author{
Brian Hung-Hin Lang, MS, FRACS and Kai Pun Wong, MBBS, MRCS \\ Division of Endocrine Surgery, Department of Surgery, Queen Mary Hospital, The University of Hong Kong, Hong Kong, \\ Hong Kong SAR, China
}

\begin{abstract}
Background. Differentiated thyroid cancer (DTC) survivors are at increased risk of developing nonsynchronous second primary malignancy (NSPM). This study aims to examine possible risk factors leading to occurrence of NSPM as well as risk factors leading to NSPM-related death in patients with DTC.

Methods. Of the 1,106 patients with DTC managed at our institution, $92(8.3 \%)$ patients developed NSPM and 40 (3.6\%) patients died of NSPM. All causes of death were confirmed by medical record, autopsy report or death certificate. Clinicopathological variables were compared between those without NSPM and with NSPM as well as between those who died of NSPM and did not die of NSPM. Significant variables on univariate analysis were entered into a Cox proportional hazards model.

Results. The median latency period from diagnosis of DTC to NSPM was 142.7 (range 16.8-511.0) months. For occurrence of NSPM, age at DTC diagnosis $\geq 50$ years old [relative risk $(R R)=2.35$ ], cumulative radioactive iodine (RAI) activity $3.0-8.9 \mathrm{GBq}(\mathrm{RR}=2.38)$, and external local radiotherapy $($ ERT $)(R R=1.95)$ were significant risk factors. For NSPM-related death, age at DTC diagnosis $\geq 50$ years old $(\mathrm{RR}=3.32)$ and nonbreast cancer $(\mathrm{RR}=5.76)$ were significant risk factors.

Conclusions. NSPM accounted for $18.7 \%$ of all deaths in DTC, but mortality was high (43.5\%). Age at DTC diagnosis $\geq 50$ years old, cumulative RAI activity 3.0-8.9 GBq, and ERT were significant risk factors for occurrence of
\end{abstract}

(C) The Author(s) 2011. This article is published with open access at Springerlink.com

First Received: 7 March 2011;

Published Online: 15 May 2011

B. H.-H. Lang, MS, FRACS

e-mail: blang@hkucc.hku.hk
NSPM, whereas age at DTC diagnosis $\geq 50$ years old and the diagnosis of nonbreast cancer were significant risk factors for NSPM-related death.

Differentiated thyroid carcinoma (DTC) accounts for over $90 \%$ of all follicular-derived thyroid malignancies and is the commonest primary endocrine-related malignancy. The age-adjusted incidence has doubled over the last 25 years in our locality, and a similar trend has been observed elsewhere. ${ }^{1,2}$ Despite the increasing trend, the disease-specific mortality remains low with overall 10-year disease-specific survival above $90 \%{ }^{3}$ As a result, the great majority of patients are expected to survive the disease and treatment, but since this disease mostly affects relatively young patients, the lifetime risk of developing a nonsynchronous second primary malignancy (NSPM) poses concerns. ${ }^{4}$ Previous studies found that, when all nonthyroidal cancer sites were considered, DTC survivors were at significantly increased risk of developing NSPM when compared with the general population. ${ }^{5-8}$ Specific cancer sites reported to have increased incidence include breast, stomach, salivary gland, colon, and bladder. ${ }^{5,9,10}$ Although the exact reason or cause for this apparent risk increase remains unclear, possible explanations include the effect of ionizing radiation from radioactive iodine (RAI) and external local radiotherapy (ERT), posttreatment surveillance bias, common environmental factors, dietary factors, and genetic predisposition., ${ }^{7,9,11,12}$ In addition to the increased risk of developing NSPM, those with NSPM were shown to have significantly worse overall survival than those without NSPM. ${ }^{8}$ Furthermore, the majority of DTC survivors with NSPM will eventually die of it. ${ }^{8}$ As a result, a compulsory surveillance program for DTC survivors has been proposed. ${ }^{5,7}$ However, it remains unknown which factors and patients are more likely to develop 
NSPM and die of NSPM. Perhaps, gaining better knowledge of these risk factors would be useful in future planning of such surveillance program. It would allow better selection or stratification of DTC survivors for cancer surveillance and would make the program more cost effective. To our knowledge, there have been no studies specifically focusing on which factors influence the risk of developing and dying of NSPM. Studies so far have concentrated mainly on risk factors for DTC development and DTC-related death and not on NSPM and related death. ${ }^{3,13,14}$ Therefore, the aims of the present study were to examine possible risk factors leading to occurrence of NSPM as well as risk factors leading to NSPM-related death in patients with DTC.

\section{PATIENTS AND METHODS}

From 1960 to 2009, 1,210 patients with DTC were managed at our institution. After excluding 104 (8.6\%) patients with clinically occult microcarcinomas, there were a total of 1,106 patients eligible for analysis. To ensure an accurate and updated follow-up status of all patients, a careful manual search of all patients' status in the territory-wide clinical management system (CMS) was performed. The CMS is a computerized database linking up all 41 public hospitals and provides inpatient medical records corresponding to over $90 \%$ of inpatient bed days in the region. ${ }^{15}$ Specific variables including latest date of follow-up or date of death, date of birth, cause of death, diagnosis date, and type of second nonthyroidal primary malignancy were recorded from the CMS. All causes of death were further confirmed by careful examination of the medical record, autopsy report, and/or death certificate. Clinicopathological data and management details relating to the DTC were prospectively collected since 1995.

The time to development of SPM was calculated from date of DTC diagnosis to diagnosis date of the second malignancy. SPM which occurred within 12 months from date of DTC diagnosis was considered synchronous and was excluded from analysis. NSPM was defined as second malignancy that occurred over 12 months after date of DTC diagnosis. For patients who developed two or more nonthyroidal primary malignancies after DTC, only the earliest occurred malignancy was recorded. Patients with history of antecedent SPM (i.e., diagnosed $>12$ months before DTC diagnosis) were excluded from the analysis. Time at risk for NSPM was computed from date of DTC to date of NSPM, date of death or date of last follow-up, whichever came first. For those with NSPM, time at risk of dying from it was computed from date of DTC to date of death from NSPM or date of last follow-up, whichever came first.

\section{DTC Treatment Protocol}

The management protocol for DTC remained unchanged throughout the study period, and details were described previously. ${ }^{16}$ Those with preoperative diagnosis of DTC were offered total or near-total thyroidectomy (i.e., less than $1 \mathrm{~g}$ of thyroid tissue remaining). Prophylactic central neck dissection was not routinely performed until 2006. Patients with one or more risk factors such as tumor size $>2 \mathrm{~cm}$, lymph node metastasis, age $>40$ years, presence of extrathyroidal extension, macroscopic postoperative residual disease in the neck, and/or distant metastasis were considered for RAI ablation 8-10 weeks after thyroidectomy by either $\mathrm{T} 4$ withdrawal or use of recombinant thyroid-stimulating hormone (TSH). Diagnostic wholebody ${ }^{131}$ I scans were performed approximately 6 months after RAI therapy. Three gigabecquerels $(\mathrm{GBq})$ or 80 millicuries (mCi) ${ }^{131} \mathrm{I}$ was administered as standard ablative dose, while subsequent RAI therapy involved $5.5 \mathrm{GBq}$ (or $150 \mathrm{mCi}$ ). Additional $5.5 \mathrm{GBq}$ RAI therapy was administered periodically at 4- to 6-month intervals until uptake was no longer visible or disease progressed despite treatment. The cumulative RAI dose or activity for each individual patient was calculated. ERT was reserved for those with extensive extrathyroidal tumor extension, incomplete resection (R2), and/or extracapsular lymph node metastasis. Although the above protocol was closely followed throughout the study period, individual patient preference was considered and respected. The present study protocol was approved by the local institutional review board.

\section{Statistical Analysis}

For comparison of dichotomous variables between groups, chi-squared tests and Fisher's exact tests were used. Mann-Whitney $U$ test was used for comparison of continuous variables between groups. Those variables with significance level $p<0.05$ on univariate analysis were entered into the multivariate analysis. Regression analysis was performed using the Cox proportional hazards model. All statistical analyses were performed using SPSS version 11.0 (SPSS, Inc., Chicago, IL, USA).

\section{RESULTS}

After median follow-up of 104.33 (range 14.1-570.75) months, there were 81 (7.3\%) who died of DTC, 57 (5.1\%) who died of nonthyroidal malignancy, and $76(6.9 \%)$ who died of a medical or natural cause. Over the same period, there were $170(15.4 \%)$ patients who developed at least one SPM. Of these, 78 patients developed SPM within 12 months of diagnosis of DTC (i.e., synchronous SPM) 
and 92 patients developed SPM more than 12 months after diagnosis of DTC. For the purpose of the analysis, the synchronous group $(n=78)$ was excluded. The median latency period from DTC to NSPM was 142.7 (range 16.8-511.0) months. Therefore, there were 936 patients who did not develop SPM (group A) and 92 who developed NSPM (group B).

Table 1 presents a comparison of demographics, period of DTC diagnosis, histological types of DTC, and stage of DTC between group A and group B. Patients in group B were significantly older at time of DTC diagnosis (48.0 versus 43.0 years, $p<0.001$ ), and there was a significantly greater proportion of patients belonging to the $\geq 50$ years old age group $(47.8 \%$ versus $34.6 \%, p<0.001)$. Sex and histological types of DTC appeared similar between the two groups. When the period of DTC diagnosis was compared, there was a significant greater proportion of patients belonging to group B before 1980 (29.3\% versus $14.6 \%, p<0.001)$. Tumor stages of DTC appeared similar between the two groups, although there was a tendency for more advanced DTC in group B. As a result, there was a significantly higher proportion of patients in group B receiving RAI therapy $(90.1 \%$ versus $72.8 \%, p=0.003$ ) and ERT (16.3\% versus $7.8 \%, p=0.005)$.
Table 2 presents the Cox proportional hazards analysis of risk factors for development of NSPM in DTC. Tumor stage of DTC was also entered into the multivariate analysis because it almost reached significance with $p=0.071$. Age $\geq 50$ years old, cumulative RAI activity 3.0-8.9, and ERT emerged as independent risk factors for development of NSPM in DTC. Both tumor stage of DTC and period of DTC diagnosis did not emerge as significant risk factors on multivariate analysis.

A further analysis was performed to evaluate which risk factors determine the risk of dying from NSPM. After median follow-up of 149.3 (range 19.6-531.1) months, there were $40(43.5 \%)$ patients who died of NSPM. As a proportion of the total number of deaths, NSPM accounted for $40 / 214$ or $18.7 \%$. Of the other 52 patients who did not die of NSPM, $37(71.2 \%)$ patients were still alive and free of DTC and NSPM, $3(5.8 \%)$ were free of DTC but not NSPM, 2 (3.8\%) were free of NSPM but not DTC, 2 (3.8\%) died of metastatic DTC, and 8 (15.4\%) died of a medical or natural cause. All these patients were considered as those who did not die from NSPM (i.e., group II). Table 3 presents a comparison of demographics, type of second primary malignancies, histology of thyroid carcinoma, and TNM stages between those who died of NSPM
TABLE 1 Comparison of demographics, period of diagnosis, histology of thyroid carcinoma, and TNM stages between those who did not develop second primary malignancy (SPM) (group A) and those who did develop nonsynchronous SPM (group B)

${ }^{\text {a }}$ Data available in 828 patients in group $\mathrm{A}$ and 81 patients in group $\mathrm{B}$

DTC differentiated thyroid carcinoma, TNM 6th edition AJCC/UICC tumor-nodemetastasis staging system Bold values mean $P<0.05$

\begin{tabular}{|c|c|c|c|}
\hline & Group A $(n=936)$ & Group B $(n=92)$ & $p$-Value \\
\hline Median age at DTC diagnosis & $43.0(7.1-90.9)$ & $48.0(12.3-89.2)$ & 0.005 \\
\hline Age at DTC diagnosis by groups (years) & & & 0.011 \\
\hline$<50$ & $6125.4)$ & $48(52.2)$ & \\
\hline$\geq 50$ & $324(34.6)$ & $44(47.8)$ & \\
\hline Sex & & & 0.158 \\
\hline Male & $186(19.8)$ & $24(26.1)$ & \\
\hline Female & $750(80.1)$ & $68(73.9)$ & \\
\hline Period of DTC diagnosis & & & $<\mathbf{0 . 0 0 1}$ \\
\hline Before 1980 & $137(14.6)$ & $27(29.3)$ & \\
\hline 1980-1999 & $413(44.1)$ & $51(55.4)$ & \\
\hline After 2000 & $386(41.2)$ & $14(15.2)$ & \\
\hline Histological type of DTC & & & 0.180 \\
\hline Papillary & $747(79.8)$ & $68(73.9)$ & \\
\hline Follicular & $189(20.2)$ & $24(26.1)$ & \\
\hline Stage of DTC by TNM & & & 0.071 \\
\hline Stage I/II & $664(70.9)$ & $5863.0)$ & \\
\hline Stage III/IV & $272(29.1)$ & $34(37.0)$ & \\
\hline Cumulative RAI activity $(\mathrm{GBq})^{\mathrm{a}}$ & & & 0.003 \\
\hline None & $225(27.2)$ & $8(9.9)$ & \\
\hline $3-8.9$ & $573(69.2)$ & $70(86.4)$ & \\
\hline$\geq 9.0$ & $30(3.6)$ & $3(3.7)$ & \\
\hline External local radiotherapy & & & 0.005 \\
\hline No & $863(92.2)$ & $77(83.7)$ & \\
\hline Yes & $73(7.8)$ & $15(16.3)$ & \\
\hline
\end{tabular}


TABLE 2 Cox proportional hazards analysis of risk factors for development of nonsynchronous second primary malignancy (NSPM) in differentiated thyroid carcinoma

$D T C$ differentiated thyroid carcinoma, $R A I$ radioactive iodine or ${ }^{131} \mathrm{I}$, TNM 6 th edition AJCC/UICC tumor-nodemetastasis staging system Bold values mean $P<0.05$

\begin{tabular}{llll}
\hline Covariate & Relative risk & $95 \%$ Confidence interval & $p$-Value \\
\hline $\begin{array}{l}\text { Age at DTC diagnosis by groups (years) } \\
\quad 1\end{array}$ & & \\
$\quad<0$ & 2.345 & $1.327-4.144$ & $\mathbf{0 . 0 0 3}$ \\
$\geq 50$ & & & \\
Period of DTC diagnosis & 1 & & 0.400 \\
Before 1980 & 1.301 & $0.705-2.398$ & 0.248 \\
1980-1999 & 1.720 & $0.685-4.317$ & \\
After 2000 & & & 0.128 \\
Stage of DTC by TNM & 1 & & \\
Stage I/II & 1.575 & $0.878-2.827$ & \\
Stage III/IV & & & $\mathbf{0 . 0 4 0}$ \\
Cumulative RAI activity (GBq) & 1 & $1.039-5.256$ & 0.328 \\
$<2.9$ & 2.383 & $0.496-8.174$ & \\
$3-8.9$ & 2.062 & & \\
$\geq 9.0$ & & $1.030-3.681$ & $\mathbf{0 . 0 4 1}$ \\
External local radiotherapy & 1 & & \\
No & 1.947 & & \\
Yes & & &
\end{tabular}

over $90 \% .^{3}$ As a result, the great majority are expected to survive their disease and treatment. One previous study has found that those who remain free of DTC recurrence have the same life expectancy as the normal population. ${ }^{17}$ Although theoretically patients who survive long enough and are cured of DTC would eventually develop or die of nonthyroidal malignancy or other unrelated causes, it is concerning to find that DTC survivors are at greater risk of developing SPM than the normal population. ${ }^{5-8,18}$ Studies have found that, when all cancer sites are considered, this increase in risk ranged between $10 \%$ and $40 \%$, depending on study design and patient selection. ${ }^{5,6,8,18}$ A meta-analysis of 70,000 DTC survivors reported a $20 \%$ increase in NSPM incidence. ${ }^{18}$ Possible contributing factors include use of RAI therapy, ERT, common environmental risk factors, genetic mutations, and surveillance bias., ${ }^{7,9,11,12}$ Since there is some evidence suggesting that this increased risk of NSPM could possibly be related to the treatment for DTC, more selective and restricted use of RAI therapy and ERT in DTC has been advocated. ${ }^{19}$ The first part of our analysis seems to support this hypothesis, with cumulative RAI activity of 3.0-8.9 GBq having an increased relative risk of 2.38 when compared with those who did not receive any RAI therapy. Furthermore, those who received ERT for DTC had increased relative risk of 1.95 when compared with those who were not exposed to ERT. Given that the decision for RAI and/or ERT was primarily based on tumor stage, it was also entered into the multivariate Cox proportional hazards analysis but did not emerge as an independent risk factor. The authors believed the reason why there was a significant difference in period of DTC diagnosis between the two groups was because of the malignancy. Despite its rising incidence, prognosis remains excellent with overall 10-year disease-specific survival of 
TABLE 3 Comparison of demographics, type of second primary malignancies, histology of thyroid carcinoma, and TNM stages between those who did die of NSPM and those who did not

\begin{tabular}{|c|c|c|c|}
\hline & Died of SPM $(n=40)$ & Did not die of SPM $(n=52)$ & $p$-Value \\
\hline Median age at DTC diagnosis (years) & $54.5(19.1-88.8)$ & $45.0(12.1-87.3)$ & $\mathbf{0 . 0 2 0}$ \\
\hline Age at DTC diagnosis by groups (years) & & & 0.227 \\
\hline$<50$ & $18(45.0)$ & $30(57.7)$ & \\
\hline$\geq 50$ & $22(55.0)$ & $22(42.3)$ & \\
\hline Median age at NSPM diagnosis (years) & $66.1(22.7-94.2)$ & $57.2(28.8-87.7)$ & 0.082 \\
\hline Median latency period from DTC to NSPM (months) & $129.6(16.8-357.97)$ & $163.4(18.9-511.0)$ & 0.431 \\
\hline Sex & & & 0.003 \\
\hline Male & $1742.5)$ & $7(13.5)$ & \\
\hline Female & $23(57.5)$ & $45(86.5)$ & \\
\hline Period of DTC diagnosis & & & 0.708 \\
\hline Before 1980 & $11(27.5)$ & $16(30.8)$ & \\
\hline 1980-1999 & $24(60.0)$ & $27(51.9)$ & \\
\hline After 2000 & $5(12.5)$ & $9(17.3)$ & \\
\hline Histological type of DTC & & & 0.244 \\
\hline Papillary & $32(80.0)$ & $36(69.2)$ & \\
\hline Follicular & $8(20.0)$ & $16(30.8)$ & \\
\hline Stage of DTC by TNM & & & 0.480 \\
\hline Stage I/II & $23(57.5)$ & $34(65.4)$ & \\
\hline Stage III/IV & $17(42.5)$ & $18(34.6)$ & \\
\hline Cumulative RAI activity $(\mathrm{GBq})^{\mathrm{a}}$ & & & 0.881 \\
\hline None & $4(10.5)$ & $4(9.3)$ & \\
\hline $3-8.9$ & $33(86.8)$ & $37(86.0)$ & \\
\hline$\geq 9.0$ & $1(2.6)$ & $2(4.7)$ & \\
\hline External local radiotherapy & & & 0.400 \\
\hline No & $8(20.0)$ & $7(13.5)$ & \\
\hline Yes & $32(80.0)$ & $45(86.5)$ & \\
\hline \multicolumn{4}{|l|}{ Type/site of NSPM ${ }^{b}$} \\
\hline Breast & $3(7.5)$ & $19(36.5)$ & 0.001 \\
\hline Colon & $7(17.5)$ & $4(7.7)$ & 0.199 \\
\hline Lung & $7(17.5)$ & $3(5.8)$ & 0.096 \\
\hline Stomach & $4(10.0)$ & $0(0.0)$ & $\mathbf{0 . 0 3 3}$ \\
\hline Liver & $3(7.5)$ & $1(1.9)$ & 0.313 \\
\hline Uterus & $2(5.0)$ & $2(3.8)$ & 1.000 \\
\hline Non-Hodgkin lymphoma & $2(5.0)$ & $1(1.9)$ & 0.578 \\
\hline
\end{tabular}

${ }^{a}$ Data available in 81 patients

${ }^{\mathrm{b}}$ Only nonsynchronous second primary malignancy with a total number $\geq 3$ are listed

DTC differentiated thyroid carcinoma, NSPM nonsynchronous second primary malignancy, TNM 6th edition AJCC/UICC tumor-nodemetastasis staging system

Bold values mean $P<0.05$

selection bias of the study, as patients with longer followup (i.e., diagnosed earlier) were more likely to develop NSPM than those with shorter follow-up. However, after adjusting for the effect of age, period of DTC diagnosis, and stage of DTC, both RAI and ERT emerged as independent factors for development of NSPM. However, unlike one previous study, our study was not able to establish a dose-effect relationship between cumulative
RAI activity and risk of NSPM development, because the relative risk was not significant in the group which had cumulative RAI activity $\geq 9.0 \mathrm{GBq} .{ }^{6}$ Perhaps, with a larger patient population, this result might have been significant, and so our study was underpowered in this respect. An alternative explanation might have been related to the dosethreshold phenomenon where the risk of RAI activity $\geq 9.0 \mathrm{GBq}$ far exceeded the dose threshold, imparting the 
TABLE 4 Cox proportional hazards analysis of risk factors for dying of nonsynchronous second primary malignancy (NSPM) in differentiated thyroid carcinoma

\begin{tabular}{lccc}
\hline Covariate & Relative risk & $95 \%$ Confidence interval & $p$-Value \\
\hline \multicolumn{2}{l}{ Age at DTC } & diagnosis by groups (years) & \\
$<50$ & 1 & & \\
$\geq 50$ & 3.321 & $1.095-5.383$ & $\mathbf{0 . 0 0 2}$ \\
Sex & & & \\
Female & 1 & $0.898-3.638$ & 0.097 \\
Male & 1.808 & & \\
Breast carcinoma as NSPM & & $\mathbf{0 . 0 0 6}$ \\
No & 1 & & \\
Yes & 0.174 & & \\
Stomach carcinoma as NSPM & & $0.050-0.608$ \\
No & 1 & $0.679-6.601$ & \\
Yes & 2.116 &
\end{tabular}

$D T C$ differentiated thyroid carcinoma, NSPM nonsynchronous second primary malignancy

Bold values mean $P<0.05$

same risk as RAI activity of 3.0-8.9 GBq. Age $\geq 50$ years old emerged as a significant risk factor for NSPM development as well as a significant independent factor for NSPM-related death. However, this was not a very surprising result, as many of the population-based studies have demonstrated a direct association between the incidence of many nonthyroidal solid malignancies and age. ${ }^{20}$ This is because cancer is a disease associated with aging and the majority of cancer diagnoses and deaths occur in the older population, particularly those aged $>65$ years old. ${ }^{20}$ The clinical relevance of this finding is that patients aged $\geq 50$ years old with newly diagnosed DTC are probably not only at increased risk of dying from DTC as previously shown but are also at increased risk of developing and dying from NSPM. ${ }^{3}$

In terms of overall survival of patients with DTC, after median follow-up of 104.3 (range 14.1-570.8) months, there were a total of 214 deaths. Of these, 81 (37.9\%) died of DTC, 57 (26.6\%) died of nonthyroidal malignancy, and $76(35.5 \%)$ died of a medical or natural cause. In other words, over a third of deaths were still related to DTC whereas only a fourth of deaths were related to nonthyroidal malignancy. If only NSPM was considered, it accounted for only $40 / 214$ or $18.7 \%$ of the total number of deaths. This might seem a relatively small proportion, but if one considers the fact that only 92 patients developed NSPM during follow-up, the overall mortality from NSPM was $40 / 92$ or $43.5 \%$ whereas the overall mortality from DTC was $81 / 1,106$ or $7.3 \%$.

Given the relatively high mortality in NSPM, the second part of our analysis sought to examine which factors might influence NSPM-related survival in DTC. As discussed earlier, age at diagnosis of DTC $\geq 50$ years old increased not only the risk of NSPM development but also the risk of dying from NSPM ( $R R=3.32,95 \%$ CI $1.10-5.38)$. This finding concurs with the experience of many nonthyroidal malignancies that increased age correlates with risk of cancerrelated death. ${ }^{20}$ However, it was interesting that the risk of dying from NSPM was significantly associated with age at DTC diagnosis and not age at NSPM diagnosis, although there was a tendency for older patients diagnosed with NSPM to die of it $(p=0.082)$. One possible explanation might have been because age at DTC diagnosis also influenced the decision for RAI and ERT, and so this might have been an added effect from RAI and ERT. Nevertheless, both cumulative RAI activity and ERT were not significant factors for NSPM-related deaths. Apart from age at DTC diagnosis, breast and stomach cancers were also significant factors influencing NSPM-related death. According to our data, the diagnosis of breast cancer had a relative protective effect regarding NSPM-related death relative to nonbreast NSPM. Putting it in another way, patients with nonbreast NSPM were at increased risk of dying from NSPM $(\mathrm{RR}=5.758,95 \%$ CI 1.655-20.15). Of the 22 patients with breast cancers, only $3(13.6 \%)$ died of it, whereas of the 4 patients with stomach cancers, all $4(100 \%)$ died of it. This could simply be related to the fact that the prognosis of breast cancer was significantly better than that of stomach cancer, despite better disease understanding and increasing treatment modalities. ${ }^{21}$ Interestingly, some studies have found that DTC survivors are at increased risk for both types of malignancy, because both the breast and stomach take up and accumulate ${ }^{131} \mathrm{I}$ and so are often exposed to a prolonged period of ionizing radiation. ${ }^{5,9}$ However, due to the relatively small number of NSPMs, our study was not able to calculate the risk of these cancers relative to the background risk in the general population. Although male sex did not reach significance on multivariate Cox proportional hazards analysis, it was somewhat interrelated with breast cancer, as only 1 of the 22 breast cancer patients was male. Nevertheless, male sex is believed to be a poor prognostic factor for both thyroidal and nonthyroidal malignancies. ${ }^{20}$

Given that age at DTC diagnosis $\geq 50$ years old, cumulative RAI activity of 3.0-8.9 GBq, and administration of ERT were independent risk factors for NSPM development, patients with one or more of these risk factors should be considered for surveillance of carcinoma of breast, colon, lung, and stomach. Regarding timing, this should start around 5-10 years after the DTC diagnosis, as the median latency period from DTC to NSPM development was 142.7 months or 11.9 years. Regular breast examination and/or mammographic screening, annual or biennial gastroscopy and colonoscopy, and chest radiography would be useful for early detection of these malignancies. However, more specific recommendations would be difficult to make 
because of the retrospective nature of the analysis and the relatively small number of NSPMs involved. Furthermore, this was a single institution's experience, and so the analysis was subjected to a certain degree of institutional and referral biases. The present study could be further improved if other risk factors such as physical activity, lifestyle exposures, and dietary factors were taken into consideration. ${ }^{22}$ Perhaps, future studies could also assess the impact of these risk factors on the risk of NSPM in DTC.

\section{CONCLUSIONS}

Although NSPM only accounted for $18.7 \%$ of all deaths in patients with DTC, the risk of dying from it was high (43.5\%). Age at DTC diagnosis $\geq 50$ years old, cumulative RAI activity of 3.0-8.9 GBq, and ERT were significant independent risk factors for development of NSPM. On further analysis of risk factors for NSPM-related death, age at DTC diagnosis $\geq 50$ years old remained a significant risk factor whereas the diagnosis of breast cancer was associated with a reduced risk of dying from NSPM.

OPEN ACCESS This article is distributed under the terms of the Creative Commons Attribution Noncommercial License which permits any noncommercial use, distribution, and reproduction in any medium, provided the original author(s) and source are credited.

\section{REFERENCES}

1. Cancer incidence and mortality in Hong Kong 1983-2006. Hong Kong Cancer Registry, Hong Kong. http://www3.ha.org.hk/ cancereg/e_stat.asp. Accessed on 15 Jan 2010.

2. Sprague BL, Warren Andersen S, Trentham-Dietz A. Thyroid cancer incidence and socioeconomic indicators of health care access. Cancer Causes Control. 2008;19:585-93.

3. Lang BH, Lo CY, Chan WF, Lam KY, Wan KY. Prognostic factors in papillary and follicular thyroid carcinoma: implications for cancer staging. Ann Surg Oncol. 2007;14:730-8.

4. Hay ID, Gonzalez-Losada T, Reinalda MS, Honetschlager JA, Richards ML, Thompson GB. Long-term outcome in 215 children and adolescents with papillary thyroid cancer treated during 1940 through 2008. World J Surg. 2010;34:1192-202.

5. Brown AP, Chen J, Hitchcock YJ, Szabo A, Shrieve DC, Tward JD. The risk of second primary malignancies up to three decades after the treatment of differentiated thyroid cancer. J Clin Endocrinol Metab. 2008;93:504-15.
6. Rubino C, de Vathaire F, Dottorini ME, Hall P, Schvartz C, Couette JE, et al. Second primary malignancies in thyroid cancer patients. Br J Cancer. 2003;89:1638-44.

7. Sandeep TC, Strachan MW, Reynolds RM, Brewster DH, Scelo G, Pukkala E, et al. Second primary cancers in thyroid cancer patients: a multinational record linkage study. J Clin Endocrinol Metab. 2006;91:1819-25.

8. Lang $\mathrm{BH}$, Lo $\mathrm{CY}$, Wong IO, Cowling BJ. Impact of second primary malignancy on outcomes of differentiated thyroid carcinoma. Surgery. 2010;148:1191-6.

9. Ronckers CM, McCarron P, Ron E. Thyroid cancer and multiple primary tumors in the SEER cancer registries. Int J Cancer. 2005; 117:281-8.

10. Nio Y, Iguchi C, Itakura M, et al. High incidence of synchronous or metachronous breast cancer in patients with malignant and benign thyroid tumor or tumor-like disorders. Anticancer Res. 2009;29:1607-10.

11. Sadetzki S, Calderon-Margalit R, Peretz C, et al. Second primary breast and thyroid cancers. Cancer Causes Control. 2003;14: 367-75.

12. Verkooijen RB, Smit JW, Romijn JA, Stokkel MP. The incidence of second primary tumors in thyroid cancer patients is increased, but not related to treatment of thyroid cancer. Eur J Endocrinol. 2006;155:801-6.

13. Wu HS, Young MT, Ituarte PH, et al. Death from thyroid cancer of follicular cell origin. J Am Coll Surg. 2000;191:600-6.

14. Eustatia-Rutten CF, Corssmit EP, Biermasz NR, Pereira AM, Romijn JA, Smit JW. Survival and death causes in differentiated thyroid carcinoma. J Clin Endocrinol Metab. 2006;91:313-9.

15. Wong IO, Chan WS, Choi S, Lo SV, Leung GM. Moral hazard or realised access to care? Empirical observation in Hong Kong. Health Policy. 2006;75:251-61.

16. Lang BH, Lo CY, Chan WF, Lam KY, Wan KY. Staging systems for papillary thyroid carcinoma: a review and comparison. Ann Surg. 2007;30:133-8.

17. Links TP, van Tol KM, Jager PL, et al. Life expectancy in differentiated thyroid cancer: a novel approach to survival analysis. Endocr Relat Cancer. 2005;12:273-80.

18. Subramanian S, Goldstein DP, Parlea L et al. Second primary malignancy risk in thyroid cancer survivors: a systematic review and meta-analysis. Thyroid. 2007;17:1277-88.

19. Cooper DS, Doherty GM, Hauger BR, Kloos RT, Lee SL, Mandel SJ, et al. Revised American Thyroid Association management guidelines for patients with thyroid nodules and differentiated thyroid cancer. Thyroid. 2009;19:1167-214.

20. Pal SK, Hurria A. Impact of age, sex, and comorbidity on cancer therapy and disease progression. J Clin Oncol. 2010;28:4086-93.

21. Rajdev L. Treatment options for surgically resectable gastric cancer. Curr Treat Options Oncol. 2010;11:14-23.

22. Wei EK, Wolin KY, Colditz GA. Time course of risk factors in cancer etiology and progression. J Clin Oncol. 2010;28:4052-57. 